\title{
Per-tiling, rep-tiling and Penrose tiling: a notion to edge cordial and cordial labeling
}

\author{
A.A. Sathakathulla \\ University of Modern Sciences, Dubai, UAE \\ E-mail: aasathak@gmail.com
}

Copyright $\odot 2015$ A.A. Sathakathulla. This is an open access article distributed under the Creative Commons Attribution License, which permits unrestricted use, distribution, and reproduction in any medium, provided the original work is properly cited.

\begin{abstract}
A fractal is a complex geometric figure that continues to display self-similarity when viewed on all scales. A simple, yet unifying method is provided for the construction of tiling by tiles obtained from the attractor of an iterated function system (IFS). This tiling can be used to extend a fractal transformation on the entire space upon which the IFS acts. There are many in this family of tiling fractals curves but for my study, I have considered each one from the above family of tiling fractals. These fractals have been considered as a graph and the same has been viewed under the scope of cordial and edge cordial labeling to apply this concept for further study.
\end{abstract}

Keywords: Tiling; Fractals; Graph Labeling; Cordial and Edge Cordial.

\section{Introduction}

Since a fractal curve is made of smaller copies of itself, it logically follows that a plane-filling fractal curve is a filled-in shape that is made of smaller filled-in shapes - identical to itself. This means that plane-filling fractal curves are tiling. Not only are they tiling, but they are recursively tiling. Mandelbrot called this "pertiling". These tiles are also examples of "rep-tiles". A rep-tile is a plane figure that tiles the plane and can be divided into several smaller copies of itself. Some fractal explorers, such as Tom Karzes [9], have designed pertilings that are a-periodic, called "Penrose Fractals." This means these tilings can extend out forever and never repeat the same pattern. One of Tom's designs is taken for study here. Penrose Fractals are further described by Bandt and Gummelt [1], and Gelbrich [5].

Many of its properties were published and discussed more by many scholars like Jeffrey Ventrella [4].. In this paper, our study is to enable the Cordial, Total cordial, Edge cordial and total edge cordial labeling for the above-mentioned tiling fractal curves. So In this study, the above-mentioned tiling fractal curves have been considered as a Graph with a number of vertices and edges. The construction of the graph is also part of the study of properties of the curve.

Furthermore, for our study, the following definitions are to be reintroduced for clear understanding of this paper (Sathakathulla, [13]).

A Graph $\mathrm{G}=\langle\mathrm{V}, \mathrm{E}, \psi\rangle$ consists of a non-empty set V called the set of nodes (points, vertices) of the graph, E is said to be the set of edges (may be empty) of the graph and $\psi$ is the mapping from the set of edges $\mathrm{E}$ to a set of the ordered or unordered pair of elements of $\mathrm{V}$. It would be convenient to write a graph $\mathrm{G}$, as $\langle\mathrm{V}$, E > or simply as $\mathrm{G}$.

A graph labeling is an assignment of integers to the vertices or edges, or both subject to certain conditions. Many types of labeling like harmonious, graceful, etc. are used by various researchers [3], [4], [6] in practice. A graph $G$ with $q$ edges is harmonious if there is an injection $\mathrm{f}$ from the vertices of $\mathrm{G}$ to the group of integers modulo $\mathrm{q}$ such that when each edge ' $x y$ ' is assigned the label $|f(x)+f(y)|(\bmod q)$, the resulting edge labels are distinct

A graph $\mathrm{G}$ with $\mathrm{q}$ edges is graceful if $\mathrm{f}$ is an injection from the vertices of $\mathrm{G}$ to the set $\mathrm{f}: \mathrm{V} \rightarrow\{0,1 \ldots \mathrm{q}\}$ such that, when each edge 'xy' is assigned the label $|f(x)-f(y)|$, the resulting edge labels are distinct. Eventually, after the introduction of the concept of cordial labeling by (I. Cahit, [2]) many researchers have investigated graph families or graphs, which admit cordial labeling with minor variations in cordial theme like product cordial labeling, total product 
cordial labeling and prime cordial labeling (F. Harary [6]). The brief summary of definitions which is useful for the present investigations is given below.

Definition 1.1 if the vertices of the graph are assigned values subject to certain conditions, then it is known as graph labeling.

For a dynamic survey on graph labeling, we refer to (J.A. Gallian, [4]). A detailed study on variety of applications of graph labeling is reported in (G. S. Bloom, [2]).

Definition 1.2 Let $G$ be a graph. A mapping $f: E(G) \rightarrow\{0,1\}$ is called binary edge labeling of $G$ and $f(e)$ is called the label of the edge e of $\mathrm{G}$ under $\mathrm{f}$.

For an edge $\mathrm{e}=\mathrm{uv}$, the induced edge labeling $\mathrm{f}^{*}: \mathrm{E}(\mathrm{G}) \rightarrow\{0,1\}$ is given by $\mathrm{f}^{*}(\mathrm{e})=|\mathrm{f}(\mathrm{u})-\mathrm{f}(\mathrm{v})|$. Let $\mathrm{vf}(0), \mathrm{vf}(1)$ be the number of vertices of $G$ having labels 0 and 1 respectively under $f$ while ef (0), ef (1) be the number of edges having labels 0 and 1 respectively under $\mathrm{f}^{*}$.

Definition 1.3 A binary vertex labeling of a graph $G$ is called a cordial labeling if $|\operatorname{vf}(0)-\operatorname{vf}(1)| \leq 1$ and $\mid$ ef $(0)-e f(1) \mid \leq$ 1. A graph $\mathrm{G}$ is cordial if it admits cordial labeling.

Definition 1.4 Let $G$ be a graph with two or more vertices then the total graph $T(G)$ of a graph $G$ is the graph whose vertex set is $V(G) \cup E(G)$ and two vertices are adjacent whenever they are either adjacent or incident in $G$.

Definition 1.5 A binary edge labeling of a graph $G$ is called an edge cordial labeling if $|v f(0)-v f(1)| \leq 1$ and $\mid$ ef $(0)-$ ef $(1) \mid \leq 1$. A graph $G$ is edge cordial if it admits cordial labeling

Definition 1.6 Cahit [2] introduced edge-cordial labeling as a binary edge labelingf: $E(G) \rightarrow\{0,1\}$, with the induced vertex labeling given by $f(v)=\sum_{u v \in E} f(u v)(\bmod 2)$ for each $v \in V$ such that $|\operatorname{ef}(0)-\operatorname{ef}(1)| \leq 1$. And $|v f(0)-v f(1)| \leq 1$, where ef(i) and $v f(i)(i=0,1)$ denote the number of edges and vertices labeled with 0 and 1 respectively.

Definition 1.7 As an extension of the above, we define a total edge-cordial labeling of a graph $\mathrm{G}$ with vertex set $\mathrm{V}$ and edge set $\mathrm{E}$ as an edge-cordial labeling such that number of vertices and edges labeled with 0 and the number of vertices and edges labeled with 1 differ by at most 1 (i.e) $\left|\left(v_{f}(0)+e_{f}(0)\right)-\left(v_{f}(1)+e_{f}(1)\right)\right| \leq 1$. A graph with a total edgecordial labeling is called a total edge-cordial graph.

The present work is focused on cordial and edge cordial labeling of above said curves at every iteration.

\section{Main results}

The construction and its properties are already well investigated by many scholars. Here, we provide the Pattern/diagram of the a rep-tile in $\mathrm{L}$ - shape, which is further known as the chair or Trominoe tiling for the need of further study.
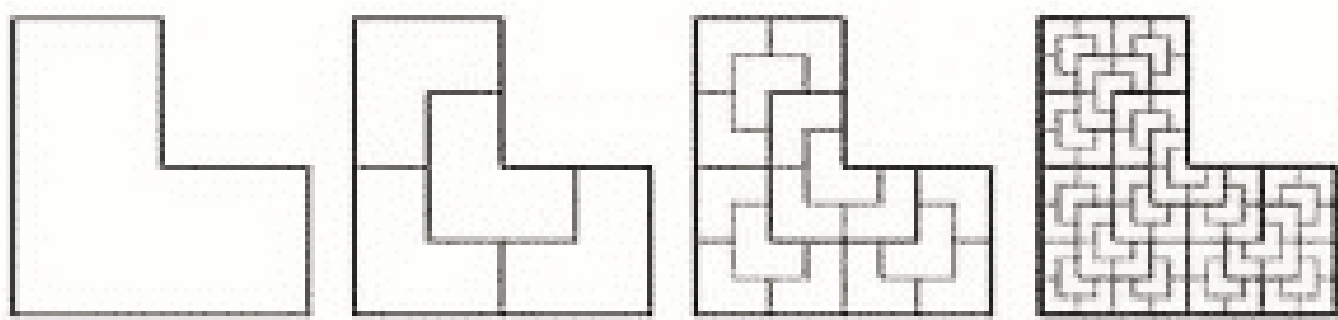

Fig. 2.1: Construction of Trominoe Rep Tile Fractal.

\subsection{Cordial labeling for Trominoe rep tiling fractal curve}

The pattern of labeling of vertices is easily understandable by seeing the figure (Fig 2.2). The vertices are numbered 0's and 1's to satisfy the condition of cordial and total cordial labeling in each iteration. It is clearly observed that all the edges having labeled 0 's are denoted by a tick mark $(\sqrt{ })$ and correspondingly all edges with 1 's are denoted by a cross $\operatorname{mark}(\mathrm{x})$. Hence it satisfies the existence of cordial labeling. In each iteration, the similar fashion of labeling is being tried to extend and is followed to check the existence of cordial labeling and total cordial labeling. Hence, the above fractal holds good for both cordial and total cordial labeling. The iteration wise number of edges and vertices are provided in the table 2.1. Every iteration values hold good for the previously mentioned definitions. But, cordial labeling is not possible for the graph with six vertices; here it is in first iteration.

\subsection{Edge cordial labeling for Trominoe rep tiling fractal curve}

Similar to the previous, the pattern of labeling of edges is easily understandable by seeing the below figure (Fig 2.3). This fractal graph is labeled with zeros and once at the edges by a tick mark $(\sqrt{ })$, a cross mark(x) at a vertices to distinguish. Furthermore, the same pattern has been extended to every iteration to fulfill the definition. The definition of edge cordial labeling is satisfying at every iteration. In addition to that, it also satisfies total edge cordial conditions. The 
results provided in the following table 2.1 hold well for all iterations. Here too, the graph with six edges fails to hold the definition of edge cordial labeling and in this case, it falls in first iteration.
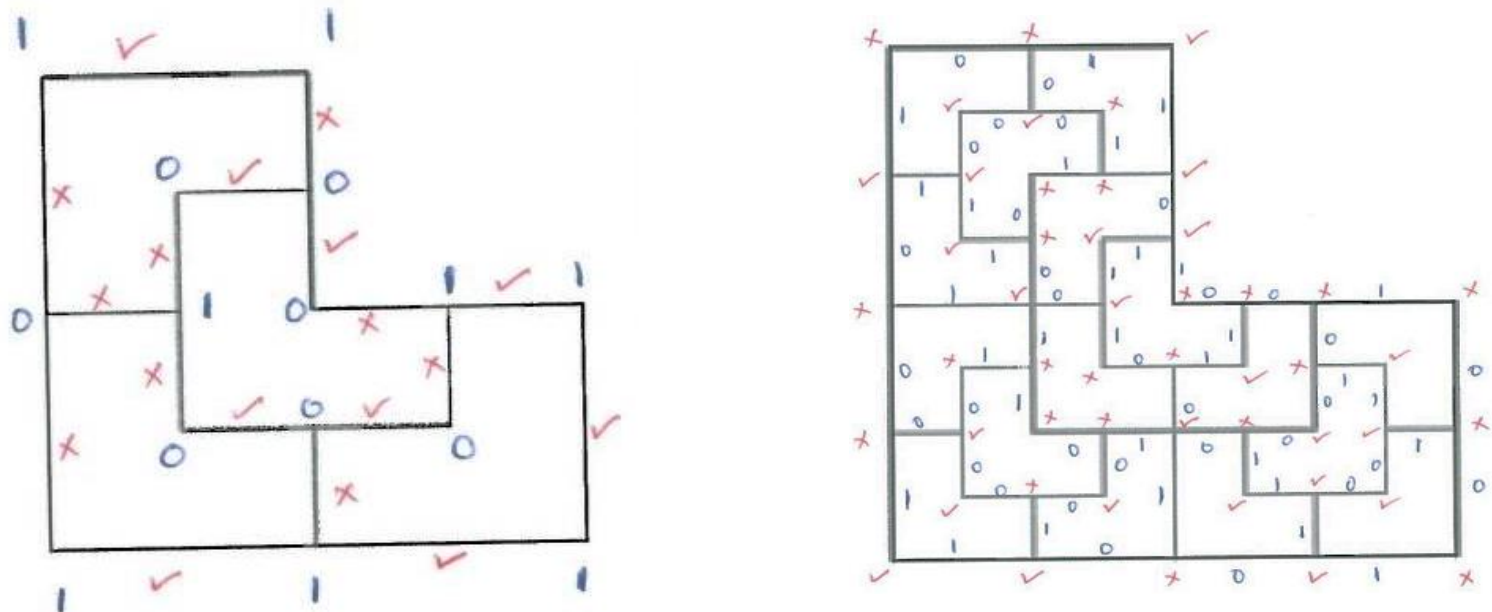

Fig. 2.2: Cordial Labeling of the Trominoe Rep Tiling Fractal Curve.
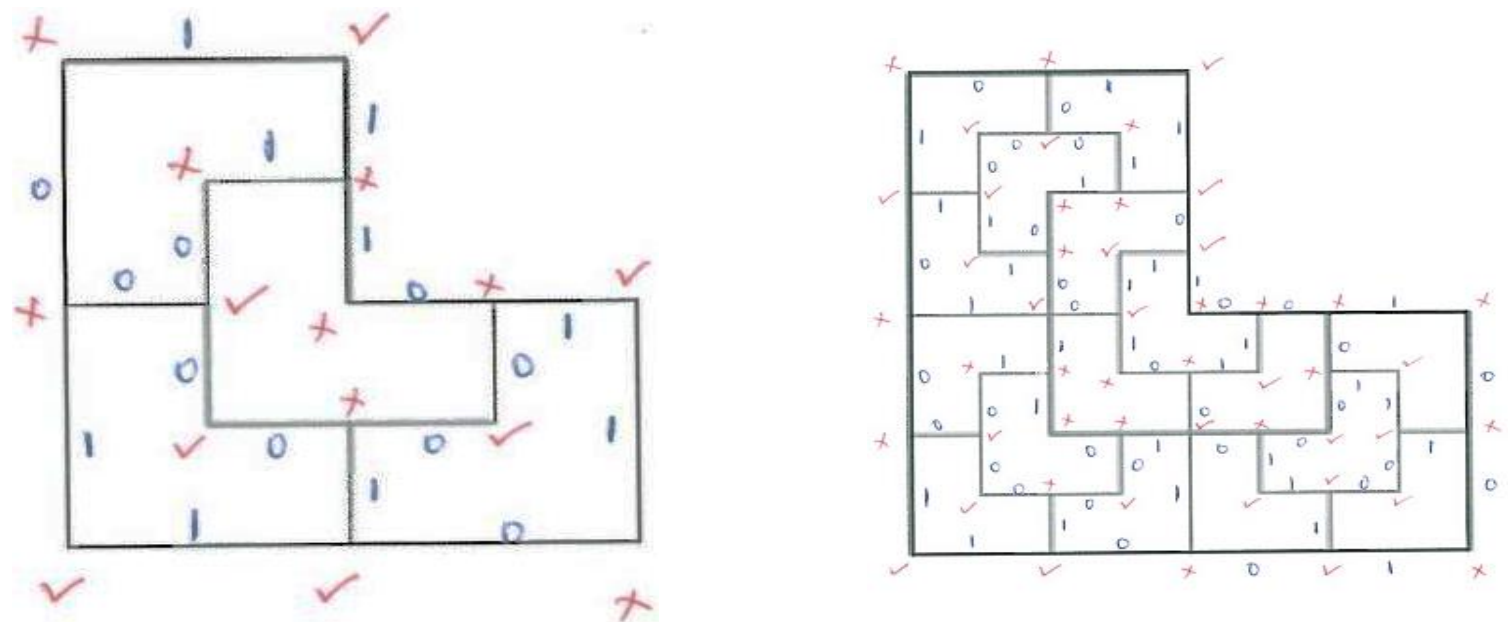

Fig. 2.3: Edge Cordial Labeling of the Trominoe Rep Tiling Fractal Curve

Table 2.1: No. of vertices and edges for Trominoe rep tiling fractal curve

\begin{tabular}{|c|c|c|c|}
\hline Iterations & No. of tiles & No. of Vertices & No. of Edges \\
\hline 1 & 1 & \multirow{2}{*}{$\begin{array}{l}|\mathrm{v}|=6 \text { but cordial not possible } \\
|\mathrm{v}|=15 \text { and }|\mathrm{vf}(0)|=8 \\
|\mathrm{vf}(1)|=7\end{array}$} & $|\mathrm{e}|=6$ but cordial not possible \\
\hline 2 & 4 & & $|e|=18$ and $|e f(0)|=|e f(1)|=9$ \\
\hline 3 & 16 & $\begin{array}{l}|v|=49 \text { and }|v f(0)|=24 \\
|v f(1)|=25\end{array}$ & $|e|=64$ and $|e f(0)|=|e f(1)|=32$ \\
\hline 4 & 64 & $\begin{array}{l}|\mathrm{v}|=177 \text { and }|\mathrm{vf}(0)|=88 \\
|\mathrm{vf}(1)|=89\end{array}$ & $|e|=240$ and $|e f(0)|=|e f(1)|=120$ \\
\hline 5 & 256 & $\begin{array}{l}|\mathrm{v}|=681 \text { and }|\mathrm{vf}(0)|=340 \\
|\mathrm{vf}(1)|=341\end{array}$ & $|e|=920$ and $|e f(0)|=|e f(1)|=460$ \\
\hline $\mathrm{n}$ & $4^{\mathrm{n}-1}$ & $\begin{array}{l}\text { Using Induction method } \\
\text { Where } I_{n-1} \text { defines previous } \\
\text { iteration's }|v| \\
\left.|v|=4 I_{n-1}-(8(n-1)-5)\right) \\
\text { for } n \geq 3 \\
\text { and }|v f(0)|+|v f(1)|=|v|\end{array}$ & $\begin{array}{l}\text { Using Induction method } \\
\text { Where } I_{n-1} \text { defines previous iteration's }|e| \\
|e|=4\left(I_{n-1}\right)-8\left(n^{2}-3 n+2\right) \text { and } \mid \text { ef }(0)|=| \text { ef } \\
\text { (1) } \mid=\frac{|\mathrm{e}|}{\mathrm{n}}\end{array}$ \\
\hline
\end{tabular}




\subsection{Half -hex rep-tiling}
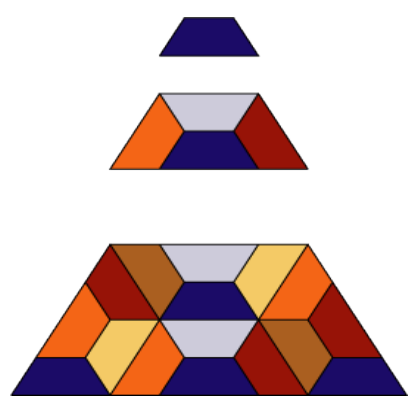

Fig. 2.4: Construction of Half-hex Tile Fractal.

This one is easily seen to be limit periodic: A large portion of the tiling is periodic. Thus it is a cut and project tiling. A detailed description of the corresponding cut and project scheme is predetermined by many scholars. The pattern of this tiling is provided in the above figure 2.4

\subsection{Cordial labeling for half-hex rep tiling fractal curve}
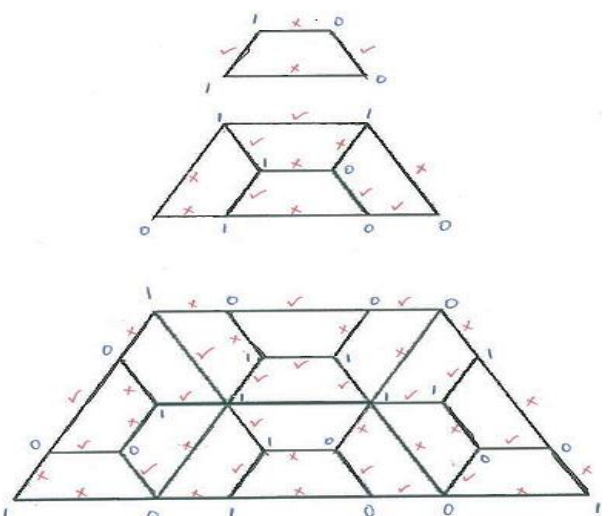

Fig: 2.5: Cordial Labeling For Half-Hex Rep Tiling Fractal Curve.

The pattern of labeling of vertices is shown in the figure (Fig 2.5). For convenience, the first three iterations are provided to add easy understanding. Like the previous, the vertices are numbered 0's and 1's to satisfy the condition of cordial and total cordial labeling in each iteration. It is clearly observed that all the edges having labeled 0's are denoted by a tick mark $(\sqrt{ })$ and correspondingly all edges with 1 's are denoted by a cross mark(x). Hence it satisfies the existence of cordial labeling and is followed to check the existence of cordial labeling and total cordial labeling. Hence, the above fractal holds good for both cordial and total cordial labeling. The iteration wise number of edges and vertices are provided in the table 2.2. Every iteration values holds good for the previously mentioned definitions.

Table 2.2: No. of vertices and edges for half hex tiling fractal curve

\begin{tabular}{|c|c|c|c|}
\hline Iterations & No. of tiles & No. of Vertices & No. of Edges \\
\hline 1 & 1 & $|\mathrm{v}|=4$ and $|\mathrm{vf}(0)|=|\mathrm{vf}(1)|=2$ & $|\mathrm{e}|=4$ and $|\mathrm{ef}(0)|=|\mathrm{ef}(1)|=2$ \\
\hline 2 & 4 & $|v|=8$ and $|v f(0)|=|v f(1)|=4$ & $\begin{array}{l}|\mathrm{e}|=11 \text { and }|\mathrm{ef}(0)|=5 \\
|\mathrm{ef}(1)|=6\end{array}$ \\
\hline 3 & 16 & $|v|=24$ and $|v f(0)|=|v f(1)|=12$ & $\begin{array}{l}|\mathrm{e}|=39 \text { and }|\mathrm{ef}(0)|=19 \\
|\mathrm{ef}(1)|=20\end{array}$ \\
\hline 4 & 64 & $|v|=78$ and $|v f(0)|=|v f(1)|=39$ & $\begin{array}{l}|\mathrm{e}|=141 \text { and }|\mathrm{ef}(0)|=70 \\
|\mathrm{ef}(1)|=71\end{array}$ \\
\hline 5 & 256 & $|\mathrm{v}|=284$ and $|\mathrm{vf}(0)|=|\mathrm{vf}(1)|=142$ & $\begin{array}{l}|e|=539 \text { and }|e f(0)|=269 \\
|e f(1)|=270\end{array}$ \\
\hline $\mathrm{n}$ & $4^{n-1}$ & $\begin{array}{l}\text { Using Induction method } \\
\text { Where } I_{n-1} \text { defines previous iteration's }|v| \\
\left.|v|=4 I_{n-1}-[10(n-1)-12)\right] \\
\text { for } n \geq 3 \\
\text { and }|v f(0)|=|v f(1)|\end{array}$ & $\begin{array}{l}\text { Using Induction method } \\
\text { Where } I_{n-1} \text { defines previous iteration's }|e| \\
\text { If } n \text { is odd } \\
|e|=4\left(I_{n-1}\right)-5(n-2) \text { and } \\
|e f(0)|+|e f(1)|=1 \text { for } n \geq 3 \\
\text { If } n \text { is even } \\
|e|=4\left(I_{n-1}\right)-5(n-1) \text { and } \\
|e f(0)|+|e f(1)|=1 \text { for } n \geq 2\end{array}$ \\
\hline
\end{tabular}




\subsection{Edge cordial labeling for half-hex rep tiling fractal curve}


Fig. 2.6: Edge Cordial Labeling For Half-Hex Rep Tiling Fractal Curve

Similar to the above, the pattern of labeling of edges is easily understandable by observing the below figure (Fig 2.6). This fractal graph is labeled with zeros and once at the edges by a tick mark $(\sqrt{ })$, a cross mark(x) at a vertices to distinguish. Furthermore, the same pattern has been extended to every iteration to fulfill the definition. The definition of edge cordial labeling is satisfying at every iteration. In addition to that, it also satisfies total edge cordial conditions. The results provided in the above table 2.2 hold well for all iterations.

\subsection{Hexagonal tiling}

A hexagon is a closed plane figure with six edges and six vertices. A regular hexagon is a convex figure with sides of the same length, and internal angles of 120 degrees. It has six rotational symmetries and six reflection symmetries. In Geometry the hexagonal tiling is a regular tiling of the Euclidean plane, in which three hexagons meet at each vertex. The following figure 2.7 shows the pattern of such tiling with coloring.

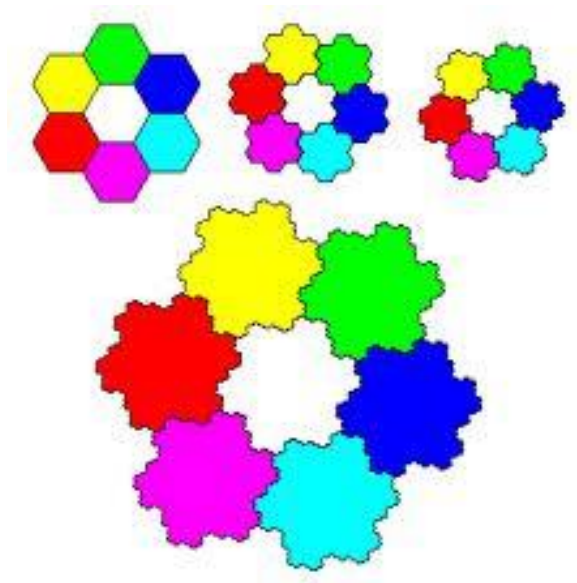

Fig. 2.7: Construction of Hexagonal Tile Fractal.

\subsection{Cordial and edge cordial labeling as of hexagonal tiling}

This tiling has six edges and six vertices in its first iteration and does not support both cordial and edge cordial labeling. However, further from the second iteration onwards the labeling of cordial and edge cordial are hold good in each iteration. The pattern of the labeling can easily be understood from the given figures. To distinguish the labeling for vertices and edges, different notations like 0's and 1's followed by a tick mark ( $\sqrt{ })$, a cross mark(x) is used. The labeling fashion is described by the given example figures for cordial and edge cordial separately. The following table 2.3 provides the number of edges and vertices for every iteration, which hold good for the mentioned definitions. 


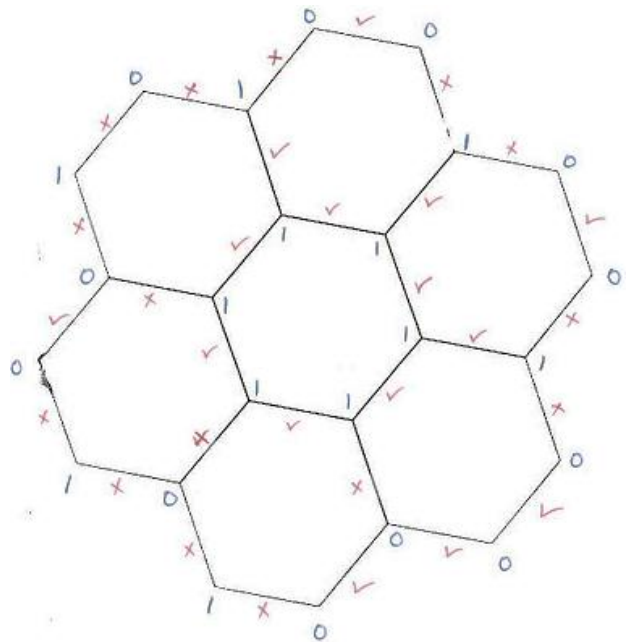

Fig: 2.8: Cordial Labeling of Hexagonal Tiling

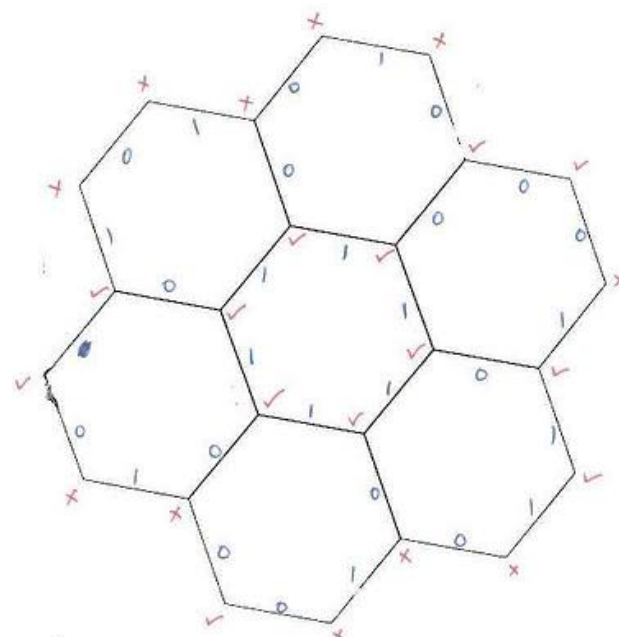

Fig. 2.9: Edge Cordial Labeling of Hexagonal Tiling

Table 2.3: No. of vertices and edges for hexagonal tiling fractal curve

\begin{tabular}{llll}
\hline Iterations & No. of tiles & No. of Vertices & No. of Edges \\
\hline 1 & 1 & $|\mathrm{v}|=6$ but cordial not possible & $|\mathrm{e}|=6$ but cordial not possible \\
2 & 7 & $|\mathrm{v}|=24$ and $|\mathrm{vf}(0)|=|\mathrm{vf}(1)|=12$ & $|\mathrm{e}|=30$ and $|\mathrm{ef}(0)|=|\mathrm{ef}(1)|=156$ \\
3 & 49 & $|\mathrm{v}|=132$ and $|\mathrm{vf}(0)|=|\mathrm{vf}(1)|=66$ & $|\mathrm{e}|=174$ and $|\mathrm{ef}(0)|=|\mathrm{ef}(1)|=87$ \\
4 & 343 & $|\mathrm{v}|=916$ and $|\mathrm{vf}(0)|=|\mathrm{vf}(1)|=408$ & $|\mathrm{e}|=1110$ and $|\mathrm{ef}(0)|=|\mathrm{ef}(1)|=555$ \\
5 & $|\mathrm{v}|=4932$ and $|\mathrm{vf}(0)|=|\mathrm{vf}(1)|=2466$ & $|\mathrm{e}|=539$ and $|\mathrm{ef}(0)|=269$ \\
& 2401 & $|\mathrm{v}|=2\left[7^{\mathrm{n}-1}+2^{2(\mathrm{n}-1)}+1\right]$ & Using Induction method \\
& & and $|\mathrm{vf}(0)|=|\mathrm{vf}(1)|=\frac{|\mathrm{v}|}{2}$ & Where $\mathrm{I}_{\mathrm{n}-1}$ defines previous iteration's $|\mathrm{e}|$ \\
$\mathrm{n}$ & $7^{\mathrm{n}-1}$ & & $|\mathrm{e}|=7\left(\mathrm{I}_{\mathrm{n}-1}\right)-6\left(3^{\mathrm{n}-3}\right)$ \\
& & & $|\mathrm{ef}(0)|=|\mathrm{ef}(1)|=\frac{\mathrm{ee} \mid}{2}$
\end{tabular}

\subsection{Penrose tiling}

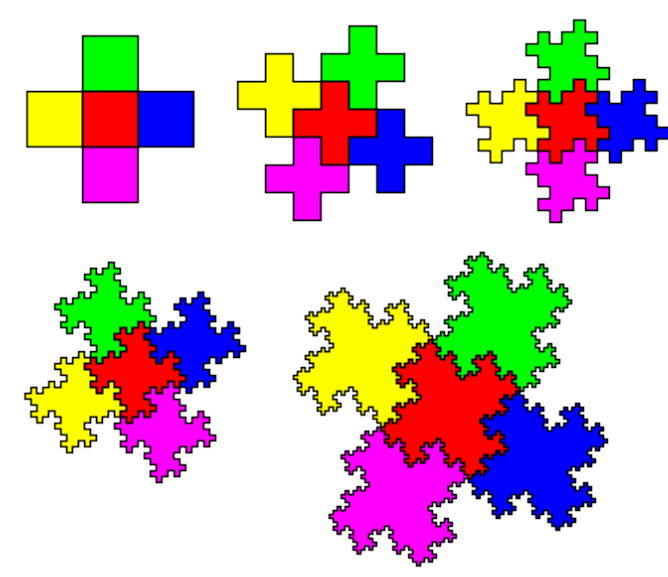

Fig. 2.10: Construction of Penrose Tiling fractal.

A Penrose tiling is a non-periodic tiling generated by an aperiodic set of prototiles. Penrose tiling is named after mathematician and physicist Roger Penrose who investigated these sets in the 1970s.

It is also developed from Greek cross. Four squares can be arranged around a central square to form a Greek cross. Greek Crosses make a lovely plane tessellation. We can try modifying a Greek cross tessellation by replacing each cross with the compound of five crosses. However, at every step, we see that the perimeter of the tiling always has twice as many crinkles as each tile. It is also called as a True rep-5 tiling. 


\subsection{Cordial and edge cordial labeling of Penrose tiling}
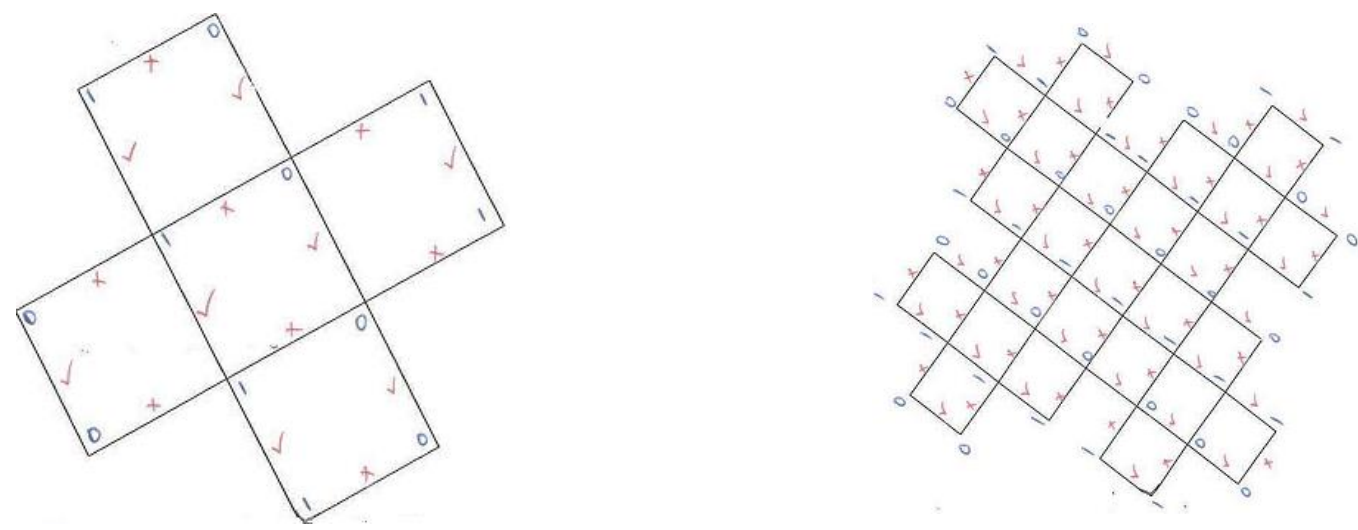

Fig: 2.10: Cordial Labeling of Penrose Tiling
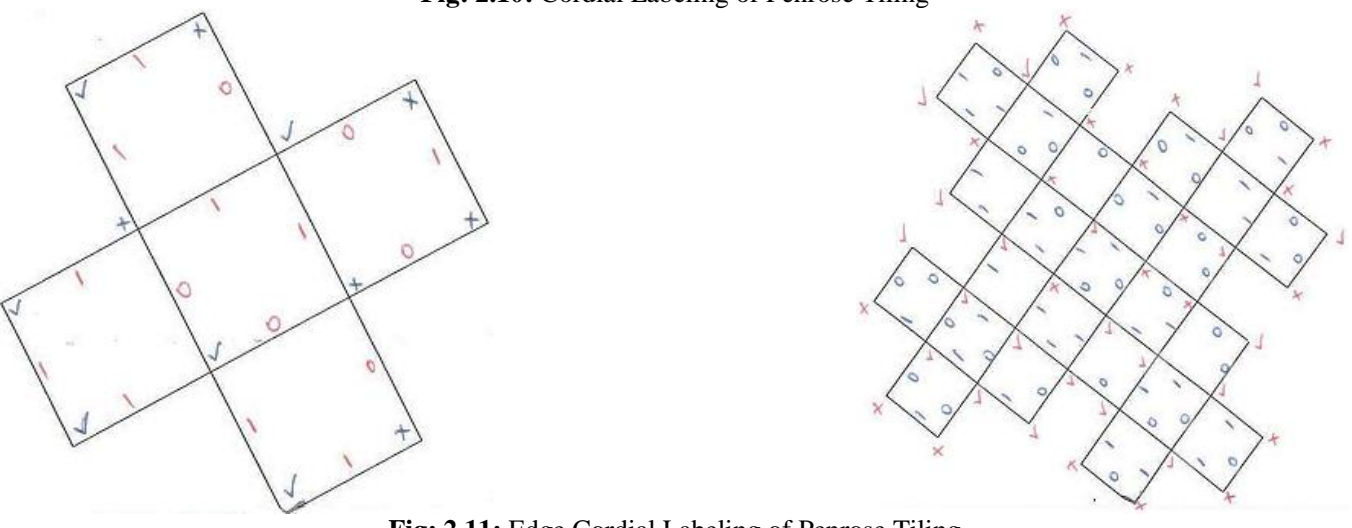

Fig: 2.11: Edge Cordial Labeling of Penrose Tiling

The labeling of Penrose tiling is easy and interesting. Since it has a number of squares, and an easy pattern of labeling in rows and columns. In cordial labeling, it is observed that, 0's and 1's are labeled alternatively. Hence, the vertical edges are getting 0 's noted by tick mark $(\sqrt{ })$, and the horizontal edges are getting 1 's noted by cross mark(x). The same fashion is continued in all the iterations. However, for edge cordial, the fashion differs due to the constraint of the definition of edge cordial, and it has been modified accordingly. However, the number of edges and vertices are held good. The results for each iteration is provided in the following table 2.4 to hold good for the mentioned definitions.

Table 2.4: No. of vertices and edges for penrose tiling fractal curve

\begin{tabular}{|c|c|c|c|}
\hline Iterations & No. of tiles & No. of Vertices & No. of Edges \\
\hline 1 & 5 & $|\mathrm{v}|=12$ and $|\mathrm{vf}(0)|=|\mathrm{vf}(1)|=6$ & $|e|=16$ and $|e f(0)|=|e f(1)|=8$ \\
\hline 2 & 25 & $|\mathrm{v}|=44$ and $|\mathrm{vf}(0)|=|\mathrm{vf}(1)|=22$ & $|\mathrm{e}|=68$ and $|\mathrm{ef}(0)|=|\mathrm{ef}(1)|=34$ \\
\hline 3 & 125 & $|\mathrm{v}|=180$ and $|\mathrm{vf}(0)|=|\mathrm{vf}(1)|=90$ & $|\mathrm{e}|=304$ and $|\mathrm{ef}(0)|=|\mathrm{ef}(1)|=152$ \\
\hline 4 & 625 & $|\mathrm{v}|=788$ and $|\mathrm{vf}(0)|=|\mathrm{vf}(1)|=394$ & $|\mathrm{e}|=1412$ and $|\mathrm{ef}(0)|=|\mathrm{ef}(1)|=706$ \\
\hline 5 & 3125 & $|v|=3612$ and $|v f(0)|=|v f(1)|=1806$ & $\begin{array}{l}|\mathrm{e}|=6736 \text { and }|\mathrm{ef}(0)|=269 \\
\operatorname{ef}(1) \mid=3368\end{array}$ \\
\hline $\mathrm{n}$ & $5^{n}$ & $\begin{array}{l}\text { Using Induction method } \\
\text { Where } I_{n-1} \text { defines previous iteration's }|v| \\
|v|=5 I_{n-1}-12\left(3^{n-2}\right)-4 \\
\text { and }|v f(0)|=|v f(1)|=\frac{|v|}{2}\end{array}$ & $\begin{array}{l}\text { Using Induction method } \\
\text { Where } \mathrm{I}_{\mathrm{n}-1} \text { defines previous iteration's }|\mathrm{e}| \\
|\mathrm{e}|=5\left(\mathrm{I}_{\mathrm{n}-1}\right)-4\left(3^{\mathrm{n}-1}\right) \\
|\mathrm{ef}(0)|=|\mathrm{ef}(1)|=\frac{|\mathrm{e}|}{2}\end{array}$ \\
\hline
\end{tabular}

\section{Conclusion}

In this paper, some of the fractal tiling graphs such as per tiling, rep tiling and Penrose's tiling are considered in our study. For instance, one in each category has been viewed in the form of a fractal graph with the concept of labeling particularly, cordial, edge cordial, and total cordial and total edge cordial concepts. And all the examples from each family of tiling are hold good for the study of cordial and edge cordial labeling. It seems to be all kinds of this tiling graph are satisfying the conditions of cordial and edge cordial for the family of Rep tiling. It is believed that this view of labeling will lead to further study of this tiling fractal graph into other types of labeling in graph theory and emerging 
application of this tiling graph curve in the field of Engineering and science. The existence of the above-mentioned labeling is proved, and the results are provided as detailed in the above tables. Hence, it is concluded that the tiling curve fractal graph is cordial, total cordial, edge cordial and total edge cordial.

\section{References}

[1] Bandt, C. and Gummelt, P. Fractal Penrose Tilings I. Construction and matching rules. Aequationes Mathematicae, vol. 53, Numbers 1-2, Feb. 1997.

[2] Bloom G. S. and Golomb S. W. (1977). Applications of numbered undirected graphs, Proc of IEEE, 65 (4), 562-570. http://dx.doi.org/10.1109/PROC.1977.10517.

[3] Cahit I. (1987). Cordial Graphs: A weaker version of graceful and harmonious Graphs, Ars Combinatoria, 23,201-207.

[4] Gallian, J. A. (2009). A dynamic survey of graph labeling, The Electronic Journal of Combinatorics, 16 , \#DS 6.

[5] Gelbrich, G. Fractal Penrose Tiles II: Tiles with fractal boundary as duals of penrose tiles. Aequationes Mathematicae, vol. 54, Numbers 1-2, Aug. 1997.

[6] Harary, F. (1972). Graph Theory, Massachusetts, Addison Wesley.

[7] James Gleick, Chaos, Vintage Publishers, 1998.

[8] Jeffrey Ventrella (2012) Brainfilling Curves - A Fractal Bestiary, Lulu.com.

[9] Karzes, T. Tiling Fractal curves published online at: http://karzes.best.vwh.net.

[10] M. Barnsley, Fractals Everywhere, Academic Press Inc., 1988.

[11] M. Seoud and A. E. I. Abdel Maqsoud, "On cordial and balanced labelings of graphs", Journal of Egyptian Math. Soc., Vol. 7, pp. 127-135, 1999.

[12] R. Devaney and L. Keen, eds., Chaos and Fractals: The Mathematics behind the Computer Graphics, American Mathematical Society, Providence, RI, 1989. http://dx.doi.org/10.1090/psapm/039.

[13] Sathakathulla A.A., Ter- dragon curve: a view in cordial and edge cordial labeling, International Journal of Applied Mathematical Research, 3 (4) (2014) 454-457. http://dx.doi.org/10.14419/ijamr.v3i4.3426.

[14] Sundaram M., Ponraj R. and Somasundram S. (2005). Prime Cordial Labeling of graphs, J.Indian Acad. Math., 27(2), 373-390. 